Proceeding Series of the Brazilian Society of Computational and Applied Mathematics

\title{
Análise de Controladores Fuzzy para Motor de Indução Aplicado na Modelagem e Simulação de Veículo Elétrico
}

\section{Paulo Roberto Ubaldo Guazzelli ${ }^{1}$}

Escola de Engenharia de São Carlos, USP, São Carlos, SP,

\section{Marcelo Suetake ${ }^{2}$}

Departamento de Engenharia Elétrica, UFSCar, São Carlos, SP

\section{Sérgio Henrique Evangelista ${ }^{3}$}

Departamento de Engenharia Mecânica, UFSCar, São Carlos, SP

Osmar Ogashawara ${ }^{4}$

Departamento de Engenharia Elétrica, UFSCar, São Carlos, SP

Resumo. Um drift buggy elétrico tracionado por um motor de indução é modelado e simulado no Simulink, da MathWorks ${ }^{\circledR}$, de modo que três controladores escalares fuzzy de velocidade são avaliados. Os resultados mostram que o controle fuzzy PD incremental modificado apresentou melhor resposta dinâmica do veículo.

Palavras-chave. Controle fuzzy, Modelagem, Motor de indução, Simulação, Veículo elétrico

\section{Introdução}

O aumento da preocupação com questões ambientais e com a qualidade de vida da população e a consequente normatização rigorosa de eficiência e emissão dos veículos proporcionou uma oportunidade para o estudo e projeto de veículos puramente elétricos (PEV - Purely Electric Vehicles) [3].

Diversos motores elétricos são usados em PEVs. Cada motor apresenta vantagens e desvantagens na sua utilização [4]. Este trabalho foca-se em estudar a utilização do Motor de Indução (MI) no processo de conversão de um veículo para propulsão elétrica, uma vez que ele equilibra boa confiabilidade com baixo custo [2].

Sendo o MI é um sistema não-linear, a investigação de técnicas de controle baseadas em sistemas fuzzy torna-se relevante, em virtude da sua capacidade de mapeamento de sistemas não-lineares e incertezas paramétricas. De acordo com [5], duas topologias se destacam: Fuzzy PD incremental (FPDInc), no qual a saída do sistema fuzzy é o incremento

\footnotetext{
${ }^{1}$ paulo.ubaldo@usp.br

${ }^{2}$ suetake@ufscar.br

${ }^{3}$ toddyprof@ufscar.br

4 osmaroga@ufscar.br
} 
da variável de atuação, e o Fuzzy PD com Integrador (FPD+I), no qual o sistema fuzzy atua em paralelo com um ramo de integração, compondo a variável de atuação. Em [7], é usado um controle Fuzzy PD incremental com modificação no cálculo da variável de atuação (FPDIncMod), a partir do uso da frequência assíncrona do MI.

\section{Propósitos}

Este trabalho visa atuar no projeto de conversão elétrica de um drift buggy. Tem-se por objetivos neste trabalho modelar e simular o veículo elétrico, avaliando o uso de três controladores escalares fuzzy de velocidade: FPD+I, FPDInc e FPDIncMod, verificando a melhor alternativa para a aplicação.

\section{Métodos}

Um PEV pode ser dividido em três sistemas principais: elétrico, mecânico e de controle. Como fonte de energia elétrica do veículo, utilizou-se na simulação duas baterias de chumbo-ácido em série, constituindo um banco com $24 \mathrm{~V}$, carga nominal de $90 \mathrm{Ah}$, e massa de $38 \mathrm{~kg}$, usando-se o modelo de [8].

Dois conversores foram modelados para acionamento do MI: um conversor elevador da tensão contínua das baterias e um inversor de frequência. Quanto ao conversor elevador, desenvolveu-se um modelo simplificado para redução do esforço computacional durante a simulação, em que a tensão desejada na saída $\left(V_{O U T}\right)$ é proporcional à tensão das baterias $\left(V_{B A T}\right)$, por um fator $\alpha$, ao mesmo tempo em que calcula a corrente drenada do banco de baterias $\left(I_{B A T}\right)$ em função da corrente drenada pelo motor $\left(I_{O U T}\right)$, por meio de $(1)$, que considera o rendimento $\eta_{C}$ do conversor.

$$
I_{B A T}=\frac{V_{O U T} \cdot I_{O U T}}{V_{B A T} \cdot \eta_{C}}=\frac{\alpha \cdot I_{O U T}}{\eta_{C}}
$$

O inversor foi modelado com uma ponte trifásica de IGBTs e diodos com componentes da biblioteca SimPowerSystems. Escolheu-se a modulação Space Vector por conseguir acionar o motor sem perda de tensão eficaz e também diminuir o número de comutações das chaves e a distorção harmônica [6]. O maior valor eficaz para tensões de linha senoidais sobre o MIT é calculado em função da tensão de saída do conversor elevador, como segue:

$$
V_{R M S}=\frac{V_{O U T}}{\sqrt{2}}
$$

Na simulação, o inversor deverá prover até $460 V_{R M S}$. Assim, por meio de (2), chega-se a um ganho elevador de 27,01. Assumiu-se uma eficiência de $90 \%$ do conversor elevador, e definiu-se a frequência da modulação igual a $2 \mathrm{kHz}$.

Em relação aos subsistemas elétricos, o motor se comporta como uma carga, requisitando corrente do circuito elétrico. Em caso de frenagem regenerativa, ocorre o processo contrário. O motor passa a fornecer corrente para o sistema, aumentando o estado de carga da bateria. Utilizou-se o modelo do MI com referencial $d q$ estacionário. O modelo fornece 
o torque eletromagnético gerador pelo motor, usado para calcular o torque aplicado no eixo mecânico de entrada da transmissão, após cálculo das perdas mecânicas do motor. Para simulação do MI, utilizou-se um preset model do MATLAB ${ }^{\circledR}$ de 5 hp/460 V/60 Hz.

Acoplado ao motor, tem-se a transmissão, que otimiza a entrega de torque do eixo do motor $\left(T_{i n}\right)$ para as rodas $\left(T_{\text {out }}\right)$, relacionando-os conforme (3), que considera o rendimento $\eta_{T}$ do sistema,

$$
\frac{T_{\text {out }}}{T_{\text {in }}}=\eta_{T} \cdot i=\eta_{T} \cdot \frac{\omega_{\text {in }}}{\omega_{\text {out }}}
$$

e o fator $i$, derivado da relação entre as velocidades angulares dos eixos de entrada e saída, sendo constante no caso de uma transmissão fixa, como a utilizada no drift buggy. Esse fator é determinado como a razão entre o número de dentes das suas engrenagens (48/10). Assim, simulou-se uma redução com fator 4,8 e eficiência assumida de $90 \%$.

A dinâmica veicular representa as forças opositoras ao movimento, podendo ser obtida a partir da $2^{\mathrm{a}}$ lei de Newton, de acordo com [3], como segue:

$$
\frac{d V}{d t}=\frac{\sum F_{t}-\sum F_{r}}{\delta M}
$$

em que a aceleração do veículo depende do somatório de forças que tracionam o veículo $\left(F_{t}\right)$ e do somatório de forças resistentes ao movimento do veículo $\left(F_{r}\right)$. Ainda, tem-se a massa $\mathrm{M}$ do veículo, e o fator adimensional $\delta$, que aumenta a massa do veículo a fim de considerar as inércias rotativas da transmissão.

Primeiramente, devem-se explicitar as forças resistentes ao movimento, a saber, três: força aerodinâmica, de rolamento e gravitacional, constituindo três parcelas, como se vê em (5).

$$
\sum F_{r}=\frac{1}{2} \rho C_{D} A_{F} \cdot V^{2}+M g f_{r} \cdot \cos (\beta)+M g \cdot \operatorname{sen}(\beta)
$$

A primeira parcela é a força aerodinâmica, que decorre do escoamento externo do ar em contato com o veículo em movimento, variando quadraticamente com a velocidade linear do carro, onde $A_{F}$ é área frontal, $C_{D}$ é o coeficiente aerodinâmico adimensional do veículo, e $\rho$ é a densidade do ar.

A segunda parcela consiste na força de rolamento, advinda da deformação dos pneus em contato com a pista, dependendo do peso do veículo (que é função da massa e da aceleração da gravidade $g$ ) e do chamado coeficiente de rolamento $\left(f_{r}\right)$. Ainda, o ângulo $\beta$ de inclinação da pista influi em seu cálculo.

Finalmente, a última parcela refere-se à força gravitacional, que surge apenas quando o veículo percorre um trecho com variações de nível, sendo a projeção de seu peso sobre o eixo longitudinal. Em uma descida, essa força age a favor do movimento.

As forças de tração podem ser resumidas em uma força $F_{T}$ que é obtida a partir do torque de saída da transmissão, em função do raio das rodas do veículo, como se vê em (6). Ainda, de acordo com [3], essa força é limitada a um valor máximo na aceleração $\left(F_{M A X}\right)$ e na frenagem $\left(F_{M I N}\right)$, em função também das dimensões da altura do Centro de Gravidade (CG) do veículo $\left(h_{G}\right)$, da distância do CG ao eixo dianteiro $\left(L_{A}\right)$ e do coeficiente de atrito da pista $(\mu)$, como se vê em $(7)$. 


$$
\begin{gathered}
\sum F_{t}=F_{T}=\frac{T_{O U T}}{R_{D}} \mid F_{T} \epsilon\left[F_{M I N}, F_{M A X}\right] \\
-\frac{\mu M g \cos (\beta)\left[L_{A}-f_{r}\left(h_{G}-R_{D}\right)\right]}{L+\mu h_{G}} \leq F_{T} \leq \frac{\mu M g \cos (\beta)\left[L_{A}-f_{r}\left(h_{G}-R_{D}\right)\right]}{L-\mu h_{G}}
\end{gathered}
$$

Ainda, pode-se calcular a máxima velocidade desenvolvida pelo carro, em $\mathrm{km} / \mathrm{h}$, por meio de (8), em função da velocidade angular nominal do motor $\left(W_{n}\right)$, em rpm, assumindo que as forças resistentes não limitem o desempenho do motor.

$$
V_{M A X}=3,6 \cdot \frac{\pi \cdot R_{D} \cdot W_{n}}{30 \cdot i}
$$

O drift buggy possui os parâmetros da Tabela 1. O coeficiente aerodinâmico e o de rolamento foram estimados conforme [3], enquanto que o coeficiente de atrito conforme [1]. A massa total foi estimada considerando a massa da estrutura, das baterias, do motor, dos componentes eletrônicos e do condutor.

Tabela 1: Parâmetros do Drift-Buggy Modelado

\begin{tabular}{|c|c|c|c|}
\hline Parâmetro & Valor & Parâmetro & Valor \\
\hline Massa total $(\mathrm{kg})$ & 199,0 & Área frontal $\left(\mathrm{m}^{2}\right)$ & 0,65 \\
\hline Fator de inércia $\delta$ & 1,1 & Coeficiente aerodinâmico & 0,70 \\
\hline Raio das rodas $(\mathrm{m})$ & 0,15 & Entre-eixos $(\mathrm{m})$ & 1,10 \\
\hline Coeficiente de rolamento dos pneus & 0,013 & Altura do CG $(\mathrm{m})$ & 0,20 \\
\hline Coeficiente de atrito dos pneus com a pista & 0,80 & Distância do eixo dianteiro ao CG $(\mathrm{m})$ & 0,77 \\
\hline
\end{tabular}

A estratégia de controle varia a amplitude e a frequência da tensão sobre o motor elétrico por meio do inversor de forma proporcional, para controle da velocidade (controle escalar $V / f$ constante). A saída do controlador é a frequência do inversor ( $f$, em Hz), que é transformada em tensão $\left(V_{R M S}\right.$, em V) por um fator de conversão, como se vê em (9). Ainda, a frequência e a tensão de acionamento são limitadas entre 10 e $100 \%$ de seus valores nominais.

$$
V_{R M S}=\frac{V_{N}}{f_{N}} \cdot f=\frac{460}{60} \cdot f=7,67 \cdot f
$$

Cada topologia de controle fuzzy calcula a variável frequência de um modo diferente. $\mathrm{O}$ controle FPDInc, mostrado na Figura 1 (a), calcula o incremento $\Delta f$ que compõe a frequência de acionamento. O controle FPDncMod, visto na figura 1 (b), adiciona também uma parcela referente à velocidade mecânica (em rpm) convertida em frequência elétrica $(\mathrm{Hz})$. Assim, o sistema fuzzy aplica uma correção na frequência de acionamento, limitada em $\pm 3 \mathrm{~Hz}$. Por fim, o controle FPD+I possui um integrador limitado em paralelo com o sistema fuzzy, como se vê na Figura 1 (c), onde a saída combinada é convertida para o domínio da variável de atuação. Os domínios de cada variável dos controladores estão na Tabela 2 . 


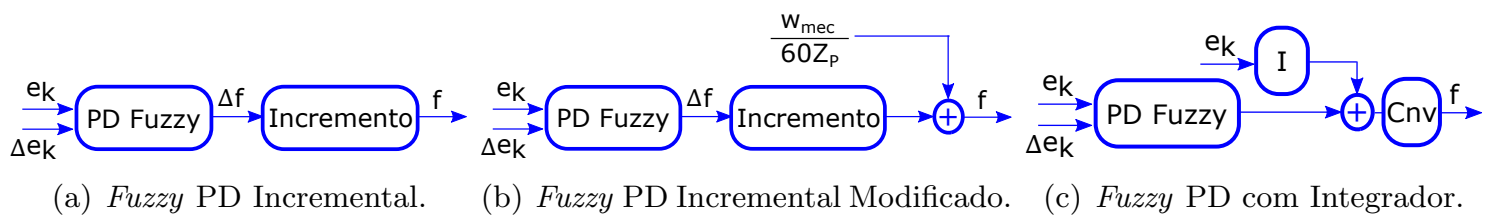

Figura 1: Estruturas dos Controladores Fuzzy.

Tabela 2: Parâmetros dos Controladores Fuzzy Avaliados.

\begin{tabular}{|c|c|c|c|}
\hline & FPDInc & FPDIncMod & FPD+I \\
\hline Domínio de $e_{k}(\mathrm{rpm})$ & {$[-500 ; 500]$} & {$[-150 ; 150]$} & {$[-5 ; 5]$} \\
\hline Domínio de $\Delta e_{k}(\mathrm{rpm})$ & {$[-0,1 ; 0,1]$} & {$[-0,07 ; 0,07]$} & {$[-0,2 ; 0,2]$} \\
\hline Domínio da saída & {$[-0,01 ; 0,01]$} & {$[-0,01 ; 0,01]$} & {$[-1 ; 1]$} \\
\hline Domínio da frequência (Hz) & \multicolumn{3}{|c|}{$[6 ; 60]$} \\
\hline Ganho de integração & - & - & 0,0011 \\
\hline
\end{tabular}

Os sistemas de Mamdani possuem funções de pertinência das entradas e da saída iguais e simétricas, conforme mostrado na Figura 2, o que reduz esforço computacional [7]. Assim, as variáveis de entrada foram normalizadas em função dos seus domínios originais para o intervalo $[-1 ; 1]$, bem como a variável de saída desnormalizada de $[-1 ; 1]$ para o seu domínio original. Têm-se os seguintes termos:\{(Negativo Grande), (Negativo Médio), (Negativo Pequeno), (Zero), (Positivo Pequeno), (Positivo Médio), (Positivo Grande)\}.

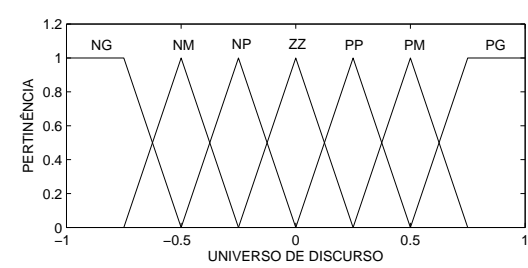

(a) Termos Linguísticos.

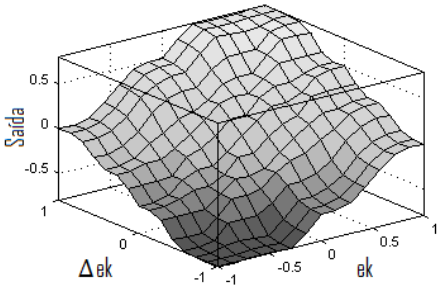

(b) Superfície de Regras.

Figura 2: Parâmetros dos Sistemas Fuzzy PD.

O modelo completo do drift buggy está na Figura 3. As baterias são conectadas ao conversor elevador, cuja saída alimenta o inversor trifásico, que aplica a tensão trifásica desejada no MI de acordo com o determinado pelo controlador fuzzy. O MI é acoplado à transmissão, que é ligada à dinâmica veicular.

Pela Equação (8), o veículo pode desenvolver até $21 \mathrm{~km} / \mathrm{h}$. Assim, ele foi simulado com referências de 10, 20 e $15 \mathrm{~km} / \mathrm{h}$ durante um segundo cada. Sendo assim, a simulação durou três segundos no total, ocorrendo em pista plana $(\beta=0 \mathrm{rad})$. Utilizou-se um passo fixo de simulação $\left(2 \cdot 10^{-5} s\right)$ e o método de integração ode3, do MATLAB ${ }^{\circledR}$. Como a referência dos controles é a velocidade angular do motor (REF), esta foi obtida a partir da velocidade linear desejada para o veículo, por meio do raio das rodas e do fator de redução. A partir da Tabela 1, chegou-se às referências para o motor de 849, 1698 e $1273 \mathrm{rpm}$. 


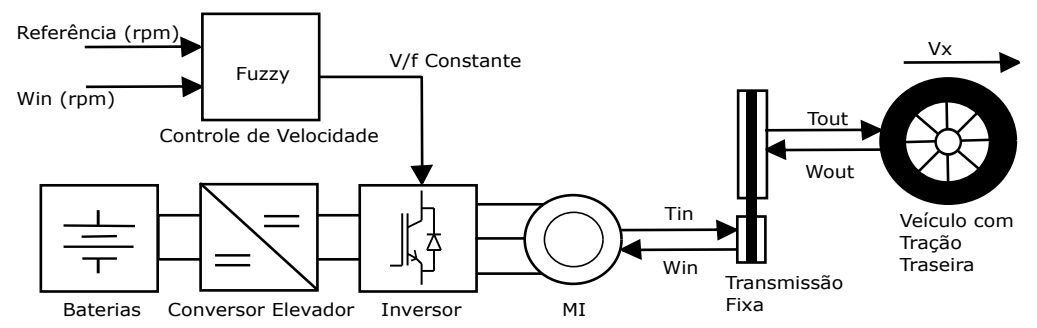

Figura 3: Diagrama de Simulação do PEV.

\section{Resultados e Discussões}

A velocidade linear do veículo ao longo do tempo pode ser vista na Figura 4 (a), para os três controladores simulados. Vê-se que em alta velocidade $(20 \mathrm{~km} / \mathrm{h})$ e durante a

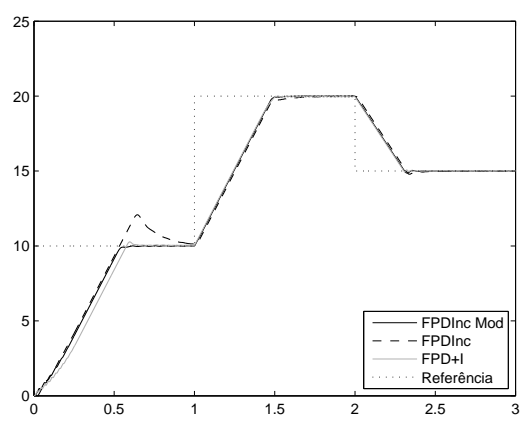

(a) Velocidade Linear do Veículo.

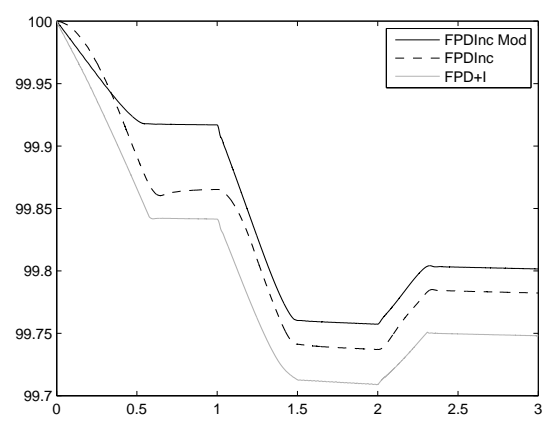

(b) Estado de Carga do Banco de Baterias.

Figura 4: Resultados Obtidos nas Simulações com Três Controladores Fuzzy.

frenagem os três controles obtiveram desempenhos semelhantes. No entanto, na velocidade de $10 \mathrm{~km} / \mathrm{h}$, percebe-se que o controle FPDInc apresentou fenômeno de windup, e que o controle FPD $+\mathrm{I}$ desenvolveu menor aceleração. Assim, o controle FPDIncMod foi menos suscetível ao windup, apresentando melhor desempenho dinâmico entre as três topologias.

Na Figura 4 (b), tem-se o gráfico do estado de carga das baterias ao longo do tempo. A análise dos três perfis de estado de carga, vistos na Figura 4 (b), mostra que o consumo do veículo com controle FPDIncMod foi menor do que com os outros dois controladores. Na última parte da simulação os três sistemas regeneraram energia para o banco de baterias, aumentando seu estado de carga, ao reduzir a velocidade do MI, caracterizando a frenagem regenerativa.

Outro ponto a ser ressaltado é o fato de o controle FPD+I utilizar um ganho de integração, cujo ajuste requer conhecimento da planta, aumentando a dependência do seu modelo, contrariando o propósito do sistema fuzzy de reduzí-la. Frente aos resultados, o sistema com controle FPDIncMod apresentou o resultado mais satisfatório, conciliando resposta dinâmica e consumo de energia. 


\section{Conclusões}

Em ambiente computacional, simulou-se um veículo elétrico movido por MI, de modo que o motor foi controlado por três topologias de sistemas fuzzy. O controle fuzzy PD incremental modificado mostrou-se melhor em relação ao desempenho mecânico do veículo e ao consumo de energia, frente as outras topologias simuladas, bem como na redução da dependência do modelo do sistema. Nas próximas etapas, destaca-se a caracterização de um MI real, para inserção dos dados na simulação, aproximando-a da prática. Para trabalhos futuros, cita-se implementar em bancada os controles de velocidade por meio de processador digital, simulando-se o perfil de torque de carga do veículo via computador, para obtenção de resultados práticos, e o desenvolvimento de controle de corrente do MI.

\section{Agradecimentos}

Os autores agradecem ao $\mathrm{CNPq}$ pelo apoio financeiro por meio de chamada pública MCTI/CNPq/SPM-PR/Petrobras n ${ }^{\circ}$ 18/2013.

\section{Referências}

[1] A. C. Canale, Automobilística: dinâmica e desempenho, Érica, (1989).

[2] D. Casadei, M. Mengoni, G. Serra, A. Tani and L. Zarri, A control scheme with energy saving and dc-link overvoltage rejection for induction motor drives of electric vehicles, IEEE Transactions on Industry Applications, vol. 46, 1436-1446, (2010), DOI: 10.1109/TIA.2010.2049627.

[3] M. Ehsani, Y. Gao and A. Emadi, Modern electric, hybrid electric, and fuel cell vehicles: fundaments, theory, and design, CRC Press, (2010).

[4] N. Hashemnia and B. Asaei, Comparative study of using different electric motors in the electric vehicles, 2008 18th International Conference on Electrical Machines, (2008).

[5] J. Jantzen, Foundations of Fuzzy Control, John Wiley and Sons, (2007).

[6] H. Pinheiro, F. Botteron, C. Rech, L. Schuch, R. F. Camargo, H. L. Hey, H. A. Gründiling e J. R. Pinheiro, Modulação space vector para inversores alimentados em tensão: uma abordagem unificada, Sba: Controle e Automação Sociedade Brasileira de Automática, vol. 16, 13-24, (2005).

[7] M. Suetake, I. N. da Silva and A. Goedtel. Embedded DSP-based compact fuzzy system and its application for induction motor V/f speed control, IEEE Transactions on Industrial Electronics, vol. 58, 750-760, (2011), DOI: 10.1109/TIE.2010.2047822.

[8] O. Tremblay and L. A. Dessaint, Experimental validation of a battery dynamic model for EV applications, World Electric Vehicle Journal, vol. 3, 1-10, (2009). 